\title{
NON-LINEAR HYSTERETIC STRUCTURAL IDENTIFICATION BY UTILIZING ON-LINE SUP- PORT VECTOR REGRESSION
}

\author{
Jian ZHANG $^{1}$ and Tadanobu SATO 2 \\ ${ }^{1}$ Student Member of JSCE, Graduate student, Dept. of Civil and Earth Resources Engineering, Kyoto University \\ (Gokasho, Uji, Kyoto 611-0011, Japan) \\ E-mail: zhangj@catfish.dpri.kyoto-u.ac.jp \\ ${ }^{2}$ Member of JSCE, Professor, Graduate School of Science and Engineering, Waseda University \\ (1-104 Totsukamachi, Shinjuku-ku, Tokyo, 169-8050, Japan) \\ E-mail: sato@catfish.dpri.kyoto-u.ac.jp
}

\begin{abstract}
Structures exhibit highly nonlinear characters under severe loads such as strong seismic excitations. Therefore, it is crucial to make nonlinear structural identification in civil engineering. However, nonlinear hysteretic structural identification is still a challenging topic due to structural model complexity and the strong noises existing in input and output (I/O) data. An efficient approach based on the incremental support vector regression (SVR) is proposed here to identify nonlinear hysteretic structural parameters on-line. Instead of the Gaussian loss function utilized in the least squares method, a novel $\varepsilon$ insensitive loss function is employed in SVR, and therefore the suggested SVR-based approach produces robust and accurate identification results. Furthermore, as an incremental algorithm employed to train SVR in a sequential way, the presented SVR-based approach not only works rapidly, but also identifys nonlinear structural constitutive parameters on-line. The performance of the proposed approach is verified by a five degree of freedom nonlinear hysteretic structural identification problem, in which two cases (power parameter is known/unknown) are both investigated. The identified results show evidently that the proposed technique has potential performance in robustness and accuracy for nonlinear structural identification, even when the measurement data in the presence of noises.
\end{abstract}

Key Words : support vector regression, on-line identification, hysteretic structure, nonlinear

\section{INTRODUCTION}

In recent years, numerous numerical methods have been developed in structural health monitoring and damage detection fields, such as statistical filter theories (Kalman') ${ }^{1)}{ }_{\infty}{ }^{2)}$ and Monte Carlo filter ${ }^{3)}$ ), Hilber Huang transforms ${ }^{4}$ etc. Structures exhibit highly nonlinear characters under severity loads such as strong seismic excitation, therefore it is critical to make nonlinear structural identification in civil engineering. A number of investigators have devoted much effort on modeling and identifying of hysteretic structural dynamic properties. The famous Bouc-Wen model is developed in 1967 to describes accurately random nonlinear vibrations of the hysteretic systems ${ }^{5), 6)}$, in which structural restoring force depends not only on the instantaneous structural response but also on the displacement history. Iwan and Gates ${ }^{7)}$ proposed a linearization scheme to estimate earthquake response of simple hysteretic structures. Villaverde and $\mathrm{Lamb}^{8}$ implemented a step-by-step integration based method to improve numerical analysis of hysteretic dynamic systems. Liu ${ }^{9)}$ utilized complex mode superposition method to solve exact solutions of nonlinear hysteretic responses of the structures subjected to arbitrary ground motions, and he employed this approach to nonlinear base-isolated structural response evaluations. The nonlinear hysteretic structural identification has a long history too ${ }^{10), 11), 12), 13)}$. Pei et al. ${ }^{14)}$ made an attempt to identify nonlinear hysteretic dynamic systems by using a powerful nonparametric identification technique (Volterra Wiener neural networks). Saadat el al. ${ }^{15)}$ presented an intelligent parameter varying (IPV) approach to identify constitutive nonlinear properties in structures subject to 
seismic excitations. Tan and Weng16) developed an iterative identification scheme to investigate the dynamic behaviour of isolated structures, in which the superstructure is chosed as a linear model and the isolation system is chosen as a bilinear hysteretic model. Benedettini et al. ${ }^{17)}$ used nonparametric identification techniques to process recorded data of nonlinear structural responses and to represent the constitutive relationship of the structure. The differential evolution algorithm was suggested by Worden et al. ${ }^{18)}$ to perform direct search of the Bouc-Wen model optimum parameters. A method based on Bayesian state estimation and bootstrap filter was presented by $\mathrm{Li}$ et al. ${ }^{19)}$ to estimate the parameters of nonlinear hysteretic system with slip on the basis of input-output data. Ni et al. ${ }^{20}$ addressed a frequency domain method to study parameter identification of friction-type hysteretic isolators based on the versatile Bouc-Wen model from the experimental data of periodic vibration tests. Some authors have presented on-line adaptive tracking technique for identification of the changes

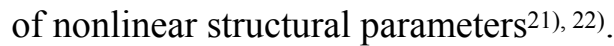

Nonlinear hysteretic structural identification is still a challenging topic in civil engineering since structural model complexity and the strong noises existing in input and output data. In this article, an efficient approach on the basis of the incremental support vector regression (SVR) is proposed to make nonlinear hysteretic structural identification accurately and robustly. SVR ${ }^{23}$ has been found work very well in many fields, and has powerful potential to be applied for system identifications. Comparing to previous nonlinear structural identification method, the proposed SVR-based approach has following features. Firstly, most articles assumed the power parameter of the Bouc-Wen model is known, as this parameter make non-liner parameter identification more intricate. In the proposed work, both power parameter known and unknown cases are investigated. Secondly, the least-squares-based identification algorithm widely utilized in the past work ${ }^{21), 22)}$ is sensitive to the outliers, in contrast, the suggested SVR-based approach has potential properties to overcome this problem and produce more accurate results, which will be described in section 2 in detail. Thirdly, most methodologies using the machine learning technologies to make system identifications only obtain "black-box" models of structures by data training. The proposed SVR-based approach not only produces the "block-box" model of structures studied, but also identifys structural parameters directly by deriving the direct relations between the training model and structural parameters.
In this work, the SVR formulations are first provided. For compariation purpose, the least squares method and least squares SVM method ${ }^{24)}$ are also given, which are helpful for us to understand the basic idea of SVR. Next, nonlinear hysteretic structural identification scheme based on the versatile Bouc-Wen model is suggested, in which two cases (power parameter is known/unknown) are both considered. An incremental SVR training algorithm are presented in the third part, which make the presented SVR-based approach not only work rapidly, but also identify structural parameters on-line. Finally, a five degree of freedom nonlinear structural system whose restoring forces are expressed by the versatile model is investigated to demonstrate the effectiveness and accuracy of the proposed SVR-based approach for nonlinear hysteretic structural identification.

\section{SVR IDEAS AND FORMULATIONS}

In this work, support vector regression (SVR) is indtroduced into the nonlinear hysteretic structural identification field. Support vector machine (SVM) is a new and promising machine learning technique. It was originally developed for classification (SVC) purposes before it was extended to regression problems (SVR) ${ }^{23), 25)}$. The classical least squares method (LS) employs Gaussian loss function, which is simple for computation but sensitive to outliers. SVR works robustly because it adopts a novel $\varepsilon$ insensitive loss function and the "mar-margin" idea. A least square version of SVM (LS-SVM) ${ }^{24}$ ) has same idea of SVM, but using Gaussian loss function instead of $\varepsilon$ insensitive loss function. It has a concise formulation and computes rapidly, but is not robust to non-Gaussian noise. A comparison for these three regression methods (LS, SVM and LS-SVM) is first provided to help us understanding the mathematic background.

\section{(1) SVR basic idea}

For linear regression problems, the robustness statistics aims at describing the structure best fitting the bulk of the empirical data

$$
y_{i}=\boldsymbol{w}^{T} \boldsymbol{x}_{i}+b+e_{i}
$$

where $\left(x_{i}, y_{i}\right)$ is a set of measurements $(i=1, \cdots, N), \boldsymbol{w}$ denotes a regression coefficient vector, $e_{i}$ is independently and identically noise, and $b$ the model offset. The objective functions of these three linear regression methods are summarized below. Their physical meanings will be interpreted later. 
LS: $\quad$ Minmize $: \sum_{i=1}^{N}\left(y_{i}-\boldsymbol{w}^{T} \boldsymbol{x}_{i}\right)^{2}$

SVM: Minmize: $\frac{1}{2}\|\mathbf{w}\|^{2}+\bar{C} \sum_{i=1}^{N}\left(\xi_{i}+\xi_{i}^{*}\right)$

LSSVM: Minmize: $\frac{1}{2}\|\boldsymbol{w}\|^{2}+\bar{C} \frac{1}{2} \sum_{i=1}^{N} e_{i}^{2}$

The classical regression approach is the least squares method that minimizes the sum of residual squares (2a). As the LS estimator is not robust to outliers. Some LS derivative methods have been developed to make robust regressions. The least median squares method aims at minimizing the least median of the squared residuals instead of the sum of them in the LS method. Therefore the LMS demonstrates enhanced performance by replacing the mean by the much less sensitive median. Another statistical technique for estimation of linear regression models is the least trimmed squares. It minimizes the trimmed sum of residuals squares and therefore features better asymptotic performance. Other robust estimators for liner regression such as S-estimators ${ }^{26)}$ can also withstand a high amount of contamination including outliers and leverage points.

Insead of the Gaussian loss function used in LS method, a novel $\varepsilon$ insensitive loss function (Fig.1) is employed in SVR. It is well known that the best loss function for estimate dependeds on the distribution of noise in $\mathrm{I} / \mathrm{O}$ measurement. Gaussian loss function is the best for unbiased estimates, while the least modulo loss function proposed is better for Laplace noise. In the real world, the noise distribution is usually unknown, and is far from both Gaussian and Laplacian noises. Therefore, Huber loss function is developed to create the best strategy for estimating functions in real-life situations. Huber loss function smoothly combines quadratic and linear functions, which is called robust loss function. Vapnik ${ }^{23}$ ) developed an $\varepsilon$ insensitive loss function that has the

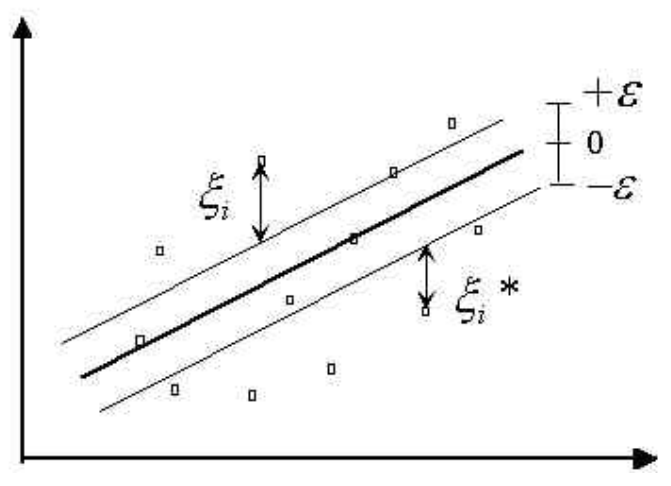

Fig.1 $\varepsilon$-insensitive loss function in SVR same structure as Huber loss function, and utilized it to produce the robust SVR machine learning technology. The $\varepsilon$ insensitive loss function (Fig.1) is

$$
L_{S V M}=\left\{\begin{array}{cc}
\mid f(x)-y) \mid-\varepsilon & \text { for } \mid f(x)-y) \mid \geq \varepsilon \\
0 & \text { otherwise }
\end{array}\right.
$$

where, $f(x)$ is the regressin function. $(x, y)$ is the observation data.

Based on this loss function, the absolute errors larger than $\varepsilon$ are accepted, while the smaller errors in the tube $(-\varepsilon, \varepsilon)$ are neglected. Practical applications of SVR illustrate that the $\varepsilon$ insensitive loss function based machine learning method works very well in many fields and shows potential robust capabilities. SVR minimizes the training error between the training data and the identified function by using the $\varepsilon$ insensitive loss function. Meanwhile, SVR adopts "Max-Margin" idea to search for an optimum hyper-plane to separate the training data into two subsets by maximizing the margin between them (Fig.2). Simply to say, SVR aims at looking for a regression function (Eq.(1) in linear regression case) to minimize the overall error expressed by Eq.(2b), in which $\xi_{i}$ and $\xi_{i}^{*}$ denote training errors in two subsets as shown in Fig.1. The first term of Eq.(2b) is from the "Max-Margin" idea of SVR, that is, to maximize the margin between two subsets. The second term minimizes the training error defined by the $\varepsilon$ insensitive loss function. $\bar{C}$ in Eq.(2b) is a parameter to trade off these two terms. Regularization method is often applied for solution of the ill-posed (instable) problem arising in the structural parameter identification. In the authors' point of view, the first term of the Eq.(2b) from the SVR "Max-Margin" idea of can be seen as the regularization term, and the parameter be seen as the regularization parameter. The relations between the SVR and the regularization theory were also discussed by Evgeniou et al. ${ }^{27)}$ in detail.

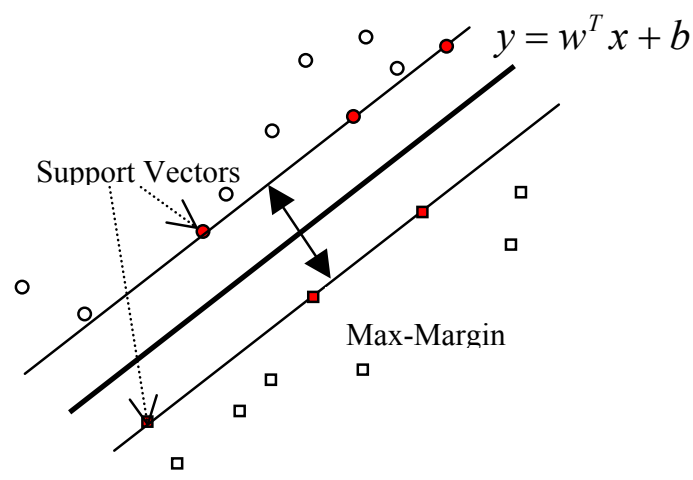

Fig.2 "Max-Margin" idea of SVR 
Suykens and Vandewalle ${ }^{24)}$ developed a least squares interpretation for SVM regression, called LS-SVM. The same idea of SVM is adopted by LS-SVM but the loss function is changed. Vapnik's $\varepsilon$ insensitive loss function is employed in SVM for the function estimation problem, while it is replaced by the classical Gaussian loss function in LS-SVM. That is, the Gaussian loss function based training error (the second term of Eq.(2c)) is minimized in LS-SVM. It has merits such as efficient computation. Meanwhile it is suffered from some drawbacks. SVR is robust in general cases because the $\varepsilon$ insensitive loss function employed, while LS-SVM only works well in Gaussian noise case, because the use of a Gaussian loss function without regularization might lead to less robust estimations.

As shown in Eq.(2), these three regression methods employ different objective functions, therefore shown different perfermonces in robustness. The LS estimator is simple but not robust to outliers. The LS-SVM works works rapidly but is sensitive to non-Gaussian noise. SVR adopts $\varepsilon$ insensitive loss function and the "mar-margin" idea, therefore it shows prosming robust capabilities. A previous work ${ }^{28)}$ compared these three methods to make linear structural identifications in details. Here we make effort to utilize SVR-based approach to make nonlinear hysteretic structural identification. A disadvantage of the SVR is that its training time increases with the square of the number of training samples and linearly with the number of variables. This shortcoming can be overcomed by the on-line training strategy which will be presented later.

\section{(2) SVR formulation}

SVR formulatons provided below are helpful for us to unkderstand and utilize SVR. Generally, there is an observed data set available for identification. For linear case, the objective of regression methods is looking for a function (Eq.(1)) to fit these data. As forgoing statement, SVR will search the optimum function by minimizing the sum error expressed in Eq.(2b). In addition, Eq.(2b) is constrained by ${ }^{23), 25)}$

$$
\left\{\begin{array}{c}
y_{i}-\mathbf{w}^{T} \boldsymbol{x}_{i}-b \leq \varepsilon+\xi_{i} \\
-y_{i}+\mathbf{w}^{T} \boldsymbol{x}_{i}+b \leq \varepsilon+\xi_{i}^{*} \\
\xi_{i}, \xi_{i}^{*} \geq 0
\end{array}\right.
$$

Namely, SVR will search for an optimum hyper-plane (defined in Eq.(1) for linear case) to separate these training data into two subsets by maximizing the margin. The regression functin Eq.(1) is produced by solving the inequality constrained optimum problem (Eqs. (2b) and (4)), which is given by the saddle point of the Lagrange function.

$$
\begin{aligned}
& L\left(\boldsymbol{w}, b, \alpha, \alpha^{*}, \eta, \eta^{*}, \xi, \xi^{*}\right)= \\
& \frac{1}{2} \sum_{k=1}^{n} w_{k}{ }^{2}+\bar{C} \sum_{i=1}^{n}\left(\xi_{i}+\xi_{i}^{*}\right) \\
&-\sum_{i=1}^{n} \alpha_{i}\left(\varepsilon+\xi_{i}-y_{i}+\boldsymbol{w}^{T} \boldsymbol{x}_{i}+b\right) \\
&-\sum_{i=1}^{n} \alpha_{i}^{*}\left(\varepsilon+\xi_{i}^{*}+y_{i}-\boldsymbol{w}^{T} \boldsymbol{x}_{i}-b\right) \\
&-\sum_{i=1}^{n}\left(\eta_{i} \xi_{i}+\eta_{i}^{*} \xi_{i}^{*}\right)
\end{aligned}
$$

where $\alpha_{i}, \alpha_{i}^{*}, \eta_{i}, \eta_{i}^{*}$ are Lagrange multipliers. Eq.(5) is transformed to the dual problem by classical Lagrange duality

$$
\max _{\alpha, \alpha^{*}, \eta, \eta^{*}} \min _{w, b, \xi, \xi^{*}} L\left(\boldsymbol{w}, b, \boldsymbol{\alpha}, \boldsymbol{\alpha}^{*}, \boldsymbol{\eta}, \boldsymbol{\eta}^{*}, \boldsymbol{\xi}, \boldsymbol{\xi}^{*}\right)
$$

So the partial derivatives of $L$ in Eq.(5) with respect to primal variables $\left(w, b, \xi_{i}, \xi_{i}^{*}\right)$ have to vanish, which leads to the Karush-Kuhn-Tucker (KKT) conditions

$$
\begin{gathered}
\frac{\partial L}{\partial \boldsymbol{w}}=\boldsymbol{w}+\sum_{i=1}^{n}\left(\alpha_{i}^{*}-\alpha_{i}\right) \boldsymbol{x}_{i}=0 \\
\frac{\partial L}{\partial b}=\sum_{i=1}^{n}\left(\alpha_{i}^{*}-\alpha_{i}\right)=0 \\
\frac{\partial L}{\partial \xi_{i}}=\bar{C}-\alpha_{i}-\eta_{i}=0 \\
\frac{\partial L}{\partial \xi_{i}^{*}}=\bar{C}-\alpha_{i}^{*}-\eta_{i}^{*}=0
\end{gathered}
$$

Substituting Eq.(7) to Eq.(6), the solution is generated by solving a optimum problem that maximizes

$$
\begin{gathered}
J=-\frac{1}{2} \sum_{i=1}^{n} \sum_{j=1}^{n}\left(\alpha_{i}-\alpha_{i}^{*}\right)\left(\alpha_{j}-\alpha_{j}^{*}\right) \boldsymbol{x}_{i}^{T} \boldsymbol{x}_{j} \\
-\varepsilon \sum_{i=1}^{n}\left(\alpha_{i}+\alpha_{i}^{*}\right)+\sum_{i=1}^{n} y_{i}\left(\alpha_{i}-\alpha_{i}^{*}\right) \\
\text { subject to: }\left\{\begin{array}{c}
\sum_{i=1}^{l}\left(\alpha_{i}-\alpha_{i}^{*}\right)=0 \\
\alpha_{i}, \alpha_{i}^{*} \in[0, C]
\end{array}\right.
\end{gathered}
$$

Formula (8) can be written as the standard quadratic problem $(\mathrm{QP})$ form that can be solved by a standard QP program. Next, the model parameter vector $\mathbf{w}$ in Eq.(1) is produced

$$
\boldsymbol{w}=-\sum_{i=1}^{n}\left(\alpha_{i}^{*}-\alpha_{i}\right) \boldsymbol{x}_{i}
$$

In brief, SVM is an exclusively data based mod- 
eling method which can be constructed solely from the multiple input-multiple outputs (MIMO) data. Eq.(8) shows that SVM can be transformed to a quadratic optimization problem. Namely, the objective function in SVM has quadratic form, which guarantees the existence of a unique global optimum and avoids the local optimum problem. Furthermore, SVR adopts the structural risk minimization (SRM) principle. Empirical risk minimization (ERM) commonly employed in statistical machine learning methods is minimizing the prediction error, whereas SRM is minimizing the upper bound of the generalization error. This feature equips SVR with a greater potential to make good predictions. In addition, SVM can describe the properties of training data by using only a few data named "Support Vectors". This is the origin of the name of SVM. For these merits, SVM has received many researchers' attentions especially in these years, and therefore it is adopted to make nonlinear hysteretic structural identification.

\section{NONLINEAR STRUCTURAL IDENTIFI- CATION SCHEME}

Structures exhibit high nonlinear characteristics under severe external forces such as strong earthquakes and winds. Numerious efforts have been paid on modeling and identifying nonlinear hysteretic structures. The versatile Bouc-Wen model is a widely accepted model originally proposed by Bouc and Wen, in which a nonlinear differential equation is developed to connect the deformation history and the the restoring force with nonlinear characteristics. The motion equation for the $n$ degree of freedom structural model with concentrated mass and nonlinear restoring force characteristic is expressed by

$$
m_{i}\left(\ddot{u}_{i}+\ddot{z}_{0}\right)+r_{i}=\left(1-\delta_{i n}\right) r_{i+1}
$$

where, $m_{i}$ and $c_{i}$, respectively, is mass and damping coefficient, $u_{i}$ is relative displacement vector to the ground, $z_{i}$ is relative displacement defined by $z_{i}=u_{i}-u_{i-1}, \ddot{z}_{0}$ is ground motion acceleration, $r_{i}$ is the nonlinear restoring force vector, and $\delta_{i n}$ Kronecker delta being 1 if $i=n$ and 0 otherwise. Based on the the versatile model, the restoring force is expressed by

$$
\dot{r}_{i}=c_{i} \ddot{z}_{i}+k_{i} \dot{z}_{i}-\alpha_{i}\left|\dot{z}_{i}\left\|\left.r_{i}\right|^{n_{i}-1} r_{i}-\beta_{i} \dot{z}_{i}\right\| r_{i}\right|^{n_{i}}
$$

Eq.(11) contains unspecified parameters. The parameters $\alpha$ and $\beta$ govern the loop shape. Power parameter $n_{i}$ controls the hysteresis loop smoothness. A variety of different hysteresis loops can be modeled by choosing these parameters suitably. The objective of this paper is identify theses structural and versatile model parameters on the basis of observation data. Structural identification algorithms often yield to nonlinear optimization problems because the structural transform equation is intrinsically expressed by a nonlinear differential equation. However, in mathematical backgrounds of nonlinear optimization problems, it is difficult to discuss generally the convergence, uniqueness and stability of solutions. So if observation equations can be expressed by linear optimization equations with respect to unknown parameters, the structural identification problem will become easier. The Bouc-Wen model with a nonlinear restoring force characteristic and the intrinsically nonlinear identification problem are considered here. The main idea is transforming the nonlinear differential equation to a linear observation equation through the high order Adam Moulton implicit scheme, next incremental SVR is employed to make nonlinear structural identification on-line.

\section{(1) Power parameter known case}

The power parameter $n_{i}$ of Bouc-Wen model is assumed as known in the first case. This assumation is often used in articles2), 21). Rearranging Eq.(11) with respect to nonlinear restoring forces $r_{i}(i=1, \cdots, n)$, the following equation is produced

$$
r_{i}=-\sum_{k=i}^{n} m_{k}\left(\ddot{z}_{0}+\ddot{u}_{k}\right)
$$

Nonlinear restoring force $r_{i}$ can be calculated from Eq.(12). Next we consider how to linearize the nonlinear identification formulation. Mathematically, there are three types of methods for solving differential equations ${ }^{29}$. The Taylor's series expansion based method is straightforward but it is difficult to deal with the higher-order derivatives of functions with lots of variables. The Runge-Kutta Methods uses evaluations of the right-hand-side function at additional points within the integration subinterval to approximate the differentials required by the Taylor's series methods. The third type are the multipoint methods such as Adam Moulton method. It uses information from subintervals prior to the solution subinterval to approximate the Taylor's series derivatives. Here, the 4-order Adam Moulton scheme (Eq.(13)) is employed to transform the nonlinear structural equation to a linear one.

$$
\frac{24}{\Delta t}\left(r_{i, k}-r_{i, k-1}\right)=9 \dot{r}_{i, k}+19 \dot{r}_{i, k-1}-5 \dot{r}_{i, k-2}+\dot{r}_{i, k-3}
$$


By assuming the power parameter $n_{i}$ is known and using the 4-order Adam Moulton scheme, the restoring force $r_{i}$ defined by a nonlinear differential equation can be rewritten in the linear form

$$
y_{i, k}=\left\{\begin{array}{c}
9 \\
19 \\
-5 \\
1
\end{array}\right\}^{T}\left[\begin{array}{c}
\boldsymbol{\Phi}_{1}{ }^{T} \\
\boldsymbol{\Phi}_{2}{ }^{T} \\
\boldsymbol{\Phi}_{3}{ }^{T} \\
\boldsymbol{\Phi}_{4}{ }^{T}
\end{array}\right]^{T}\left\{\begin{array}{c}
c_{i} \\
k_{i} \\
\alpha_{i} \\
\beta_{i}
\end{array}\right\}
$$

where,

$$
\begin{aligned}
& \boldsymbol{\Phi}_{1}=\left\{\begin{array}{c}
\ddot{z}_{i, k} \\
\ddot{z}_{i, k-1} \\
\ddot{z}_{i, k-2} \\
\ddot{z}_{i, k-3}
\end{array}\right\}, \quad \boldsymbol{\Phi}_{3}=\left\{\begin{array}{ccc}
-\left|\dot{z}_{i, k}\right|\left|r_{i, k}\right|^{n_{i}-1} & r_{i, k} \\
-\left|\dot{z}_{i, k-1}\right|\left|r_{i, k-1}\right|^{n_{i}-1} & r_{i, k-1} \\
-\left|\dot{z}_{i, k-2}\right|\left|r_{i, k-2}\right|^{n_{i}-1} & r_{i, k-2} \\
-\left|\dot{z}_{i, k-3}\right|\left|r_{i, k-3}\right|^{n_{i}-1} & r_{i, k-3}
\end{array}\right\} \\
& \boldsymbol{\Phi}_{2}=\left\{\begin{array}{c}
\dot{z}_{i, k} \\
\dot{z}_{i, k-1} \\
\dot{z}_{i, k-2} \\
\dot{z}_{i, k-3}
\end{array}\right\}, \quad \boldsymbol{\Phi}_{4}=\left\{\begin{array}{c}
-\dot{z}_{i, k}\left|r_{i, k}\right|^{n_{i}} \\
-\dot{z}_{i, k-1}\left|r_{i, k-1}\right|^{n_{i}} \\
-\dot{z}_{i, k-2}\left|r_{i, k-2}\right|^{n_{i}} \\
-\dot{z}_{i, k-3}\left|r_{i, k-3}\right|^{n_{i}}
\end{array}\right\}
\end{aligned}
$$

Up to now, the nonlinear structural identification results can be generated by solving a liner problem when the structural responses are measured, because the nonlinear constitutive parameters $c_{i}, k_{i}, \alpha_{i}$ and $\beta_{i}$ except $n_{i}$ appeared in Eq.(14) as linear combination. Note that Eq.(14) has a same form with Eq.(1), in which $y(t)$ is calculated from the restrong forces, $\boldsymbol{x}$ is measurement matrix, and $\boldsymbol{w}$ consists unknown structural parameters. Therefore, the forgoing SVR formulatins can be utilized to solve the linear observation equation with respect to unknown nonlinear constitutive parameters.

\section{(2) Power parameter unknown case}

This power parameter of the versatile model $n_{i}$ is assumed to be known in the first case, because this parameter can not be linearized in the observation equation. A strategy to overcome this shortcoming is assuming some values for the unknown $n_{i}$ parameter, next identifying which value matchs well with the true $n_{i}$ value. For instance, by assuming $n_{i}$ equals to integers from 1 to $m$, the restoring force $r_{i}$ defined in Eq.(11) is rewritten as

$$
\begin{aligned}
\dot{r}_{i}= & c_{i} \ddot{z}_{i}+k_{i} \dot{z}_{i}-\alpha_{i 1}\left|\dot{z}_{i}\right| r_{i} \cdots-\beta_{i 1} \dot{z}_{i} \| r_{i} \mid \\
& -\alpha_{i m}\left|\dot{z}_{i}\left\|\left.r_{i}\right|^{m-1} r_{i} \cdots-\beta_{i m} \dot{z}_{i}\right\| r_{i}\right|^{m}
\end{aligned}
$$

In a similar way as derivations of Eq.(14), the structural nonlinear equation is linearized by the 4-order Adam Moulton equation,

$$
y_{i, k}==\left\{\begin{array}{c}
9 \\
19 \\
-5 \\
1
\end{array}\right\}^{T}\left[\begin{array}{c}
\boldsymbol{\Phi}_{1}{ }^{T} \\
\boldsymbol{\Phi}_{2}{ }^{T} \\
\vdots \\
\boldsymbol{\Phi}_{2 j+1}{ }^{T} \\
\boldsymbol{\Phi}_{2 j+2}{ }^{T} \\
\vdots \\
\boldsymbol{\Phi}_{2 m+1}{ }^{T} \\
\boldsymbol{\Phi}_{2 m+2}{ }^{T}
\end{array}\right]^{T}\left\{\begin{array}{c}
c_{i} \\
k_{i} \\
\alpha_{i} \\
\beta_{i}
\end{array}\right\}
$$

where, $\boldsymbol{\Phi}_{1}$ and $\boldsymbol{\Phi}_{2}$ are same as that in Eq.(14), $\boldsymbol{\Phi}_{2 j+1}$ and $\boldsymbol{\Phi}_{2 j+2}(j=1, \cdots, m)$ have similar forms as $\boldsymbol{\Phi}_{3}$ and $\boldsymbol{\Phi}_{4}$ with $j$ instead of $n_{i}$.

In ideal free noise conditions, the identified parameters $\alpha_{i}$ and $\beta_{i}$ corresponding to the true $n_{i}$ values should have relative large values, while those corresponding to false $n_{i}$ values will vanish. Therefore, once parameters $\alpha_{i}$ and $\beta_{i}(i=1, \cdots, m)$ are generated, the $n_{i}$ value is identified indirectly. More accurate identified parameters can be produced by subdividing the assumed $n_{i}$ interval values.

\section{ON-LINE SVR FOR STRUCTURAL IDENTIFICATION}

As described in last section, structural motion equations can be transformed to the linear form. Therefore nonlinear structural identification problem becomes an easier line regression problem, which can be solved by SVR robustly. However, conventional SVR suffer to the drawback of time consuming, because it uses a QP optimization as an inner loop. In addition, conventional SVR cannot make function estimations on-line. Due to these reasons, it is necessary to study the incremental algorithm to train SVR in a sequential way. Vapnik ${ }^{23}$ developed a chunking method to solve the SVM QP problem. Osuna et al. ${ }^{30)}$ presented a novel decomposition algorithm training SVM over large data sets. The famous sequential minimal optimization (SMO) training algorithm for SVM was suggested by Platt ${ }^{31}$ ) in 1998. SMO breaks the large QP problem into a sequence of smallest possible QP problems that can be solved analytically. Flake and Lawrence ${ }^{32)}$ improved the SMO training to SVM regression. Through these methods, the SVR training is faster because large scale QP problem is divided into several smaller QP or solved analytically. However, all the described algorithms cannot be executed on-line. Based on the incremental and decremental theory developed by Cauwenberghs and Poggio ${ }^{33)}$. Ma et al. ${ }^{34)}$ suggested an accurate on-line recursive algorithm for training SVR. A similar work is done by 
Martin $^{35)}$. Next, the recursive algorithm for on-line SVR training is concisely provided based on the SVR throry (Eqs. (4)-(9)). A more detailed version of the on-line SVR theory presentation can be found in $\mathrm{Ma}$ et al. ${ }^{34)}$ and Martin ${ }^{35}$. The provided formulations will be used to train SVR and make nonlinear hysteretic structural identification on-line for numerical examples in next section.

The main idea of on-line SVR is using a recursive algorithm to efficiently update a trained SVR function whenever a sample $\boldsymbol{x}_{c}$ is added to the training set. Namely, a recursive algorithm is developed to change the Lagrange multipliers corresponding to the new sample $\boldsymbol{x}_{c}$ in a finite number of discrete steps until it meets the KKT conditions, while ensuring that the existing samples continue to satisfy the KKT conditions at each recursive step. As forgoing statement, the solution for SVR formulation is given by the saddle point of the Lagrange function show in Eq.(5). The partial derivatives of $L$ with respect to the primal variables $\left(\boldsymbol{w}, b, \xi_{i}, \xi_{i}^{*}\right)$ have to vanish, which leads to the KKT conditions (Eq.(7)). Based on the KKT conditions, the training data are divided to several sets

$$
\left\{\begin{array}{ccc}
h\left(\boldsymbol{x}_{i}\right)>\varepsilon & \boldsymbol{\theta}_{i}=-\bar{C} & i \in \boldsymbol{E} \\
h\left(\boldsymbol{x}_{i}\right)=\varepsilon & -\bar{C}<\boldsymbol{\theta}_{i}<0 & i \in \boldsymbol{S} \\
-\varepsilon<h\left(\boldsymbol{x}_{i}\right)<\varepsilon & \boldsymbol{\theta}_{i}=0 & i \in \boldsymbol{R} \\
h\left(\boldsymbol{x}_{i}\right)=-\varepsilon & 0<\boldsymbol{\theta}_{i}<\bar{C} & i \in \boldsymbol{S} \\
h\left(\boldsymbol{x}_{i}\right)<-\varepsilon & \boldsymbol{\theta}_{i}=\bar{C} & i \in \boldsymbol{E}
\end{array}\right.
$$

where $\boldsymbol{E}$ called error support vectors, $\boldsymbol{S}$ is margin support vectors, and $\boldsymbol{R}$ remaining samples. A coefficient and a function corresponding to the $i$ sample $\boldsymbol{x}_{i}$ are first defined

$$
\boldsymbol{\theta}_{i}=\boldsymbol{\alpha}_{i}-\boldsymbol{\alpha}_{i}^{*}, h\left(\boldsymbol{x}_{i}\right)=f\left(\boldsymbol{x}_{i}\right)-y_{i}
$$

In SVM, all $\theta$ corresponding to the training samples need to satisfy the KKT conditions. The solutions for on-line SVR have to keep this condition too. After a new data $\boldsymbol{x}_{c}$ is added in the training data set of SVR, a recursive algorithm is employed to find an optimum $\boldsymbol{\theta}_{c}$, that is, $\boldsymbol{\theta}_{c}$ is updated in each recursive step by the magnitude $\Delta \boldsymbol{\theta}_{c}$ until it satisfy the KKT conditions. Meanwhile, the coefficient $\boldsymbol{\theta}_{i}$ for each old training data is updated in each step also to still keep the KKT conditions. Therefore the relations between the improvement $\Delta \boldsymbol{\theta}_{c}$ of the new training data and those of the old training data need be studied, which are shown as

$$
\left[\begin{array}{cccc}
0 & 1 & \cdots & 1 \\
1 & \boldsymbol{q}_{s_{1} s_{1}} & \cdots & \boldsymbol{q}_{s_{1} s_{l s}} \\
\vdots & \vdots & \ddots & \vdots \\
1 & \boldsymbol{q}_{s_{s} s_{1}} & \cdots & \boldsymbol{q}_{s_{s s} s_{l s}}
\end{array}\right]\left[\begin{array}{c}
\Delta b \\
\Delta \boldsymbol{\theta}_{s_{1}} \\
\vdots \\
\Delta \boldsymbol{\theta}_{s_{l s}}
\end{array}\right]=-\left[\begin{array}{c}
1 \\
\boldsymbol{q}_{s_{1} c} \\
\vdots \\
\boldsymbol{q}_{s_{l s} c}
\end{array}\right] \Delta \boldsymbol{\theta}_{c}
$$

where $\boldsymbol{q}_{i j}=\boldsymbol{x}_{i}{ }^{T} \boldsymbol{x}_{j} . \boldsymbol{S}=\left\{\begin{array}{lll}s_{1}, & s_{2}, \cdots, & s_{l s}\end{array}\right\}$ denotes the samples in the $\boldsymbol{S}$ set. Define $\boldsymbol{N}=\boldsymbol{E} \cup \boldsymbol{R}=\left\{n_{1}, n_{2}, \cdots, n_{\text {ln }}\right\}$ to denote the Non- $\boldsymbol{S}$ samples. The equation to update $h(\boldsymbol{x})$ can be derived

$$
\left[\begin{array}{c}
\Delta h\left(\boldsymbol{x}_{n_{1}}\right) \\
\Delta h\left(\boldsymbol{x}_{n_{1}}\right) \\
\vdots \\
\Delta h\left(\boldsymbol{x}_{n_{\mathrm{ln}}}\right)
\end{array}\right]=\left(\left[\begin{array}{c}
\boldsymbol{q}_{n_{1} c} \\
\boldsymbol{q}_{n_{2} c} \\
\vdots \\
\boldsymbol{q}_{n_{\mathrm{ln}} c}
\end{array}\right]-\boldsymbol{\gamma}\left[\begin{array}{c}
1 \\
\boldsymbol{q}_{s_{1} c} \\
\vdots \\
\boldsymbol{q}_{s_{l s} c}
\end{array}\right]\right) \Delta \boldsymbol{\theta}_{c}
$$

where,

$$
\boldsymbol{\gamma}=\left[\begin{array}{cccc}
1 & \boldsymbol{q}_{n_{1} s_{1}} & \cdots & \boldsymbol{q}_{n_{1} s_{l s}} \\
1 & \boldsymbol{q}_{n_{2} s_{1}} & \cdots & \boldsymbol{q}_{n_{2} s_{s s}} \\
\vdots & \vdots & \ddots & \vdots \\
1 & \boldsymbol{q}_{n_{\mathrm{ln}} s_{1}} & \cdots & \boldsymbol{q}_{n_{\mathrm{ln}} s_{l s}}
\end{array}\right]\left[\begin{array}{cccc}
0 & 1 & \cdots & 1 \\
1 & \boldsymbol{q}_{s_{1} s_{1}} & \cdots & \boldsymbol{q}_{s_{1} s_{l s s}} \\
\vdots & \vdots & \ddots & \vdots \\
1 & \boldsymbol{q}_{s_{l s} s_{1}} & \cdots & \boldsymbol{q}_{s_{l s} s_{l s s}}
\end{array}\right]^{-1}
$$

Given $\Delta \boldsymbol{\theta}_{c}$, all the $\boldsymbol{\theta}_{i}$ and $h\left(\boldsymbol{x}_{i}\right)$ can be updated as follows:

- For $i \in \boldsymbol{S}, \boldsymbol{\theta}_{i}$ can be updated based on given $\Delta \boldsymbol{\theta}_{c}$ and Eq.(19). $h\left(\boldsymbol{x}_{i}\right)$ is constant as shown in Eq.(17) if the $S$ set stays unchanged.

- For $i \in \boldsymbol{N}, h\left(\boldsymbol{x}_{i}\right)$ can be updated based on given $\Delta \boldsymbol{\theta}_{c}$ and Eq.(20). $\boldsymbol{\theta}_{i}$ is constant as suggested by Eq.(17) if the $\boldsymbol{S}$ set stays unchanged.

The procedures provided below help us to find an appropriate $\Delta \boldsymbol{\theta}_{c}$ to finish the incremental SVR algorithm.

1. set $\boldsymbol{\theta}_{c}=0$, and calculate $h\left(\boldsymbol{x}_{c}\right)=f\left(\boldsymbol{x}_{c}\right)-y_{c}$.

2. if $\left|h\left(\boldsymbol{x}_{c}\right)\right|<\varepsilon$, assign $\boldsymbol{x}_{c}$ to $\boldsymbol{R}$, and exit.

3. if $\left|h\left(\boldsymbol{x}_{c}\right)\right|<\varepsilon$, determined the allowed $\Delta \boldsymbol{\theta}_{c}$ for each sample according to Eq.(19). The largest $\Delta \boldsymbol{\theta}_{c}$ is taken as the one with the minimum absolute value among all the possible $\Delta \boldsymbol{\theta}_{c}$.

4. increment $\boldsymbol{\theta}_{c}$ with a largest $\Delta \boldsymbol{\theta}_{c}$, and update $\boldsymbol{\theta}_{k}$ $k \in \boldsymbol{S}$ and $h\left(\boldsymbol{x}_{l}\right) l \notin \boldsymbol{S}$ until the new sample $\boldsymbol{x}_{c}$ meets the KKT conditions, and the old samples still meet the KKT conditions.

In brief, the adopted on-line algorithm permits efficient retraining SVR when a new sample $\boldsymbol{x}_{c}$ is added to the training set. The basic idea is to update the coefficient $\boldsymbol{\theta}_{c}$ corresponding to the new sample $\boldsymbol{x}_{c}$ in a finite number of discrete steps until it meets the KKT conditions, while ensuring that the existing 
samples continue to satisfy the KKT conditions at each step. The relation between the change of $\boldsymbol{\theta}_{c}$, and the change of other coefficients is first given (Eq.(19)), then a procedure to determine the largest allowed $\Delta \boldsymbol{\theta}_{c}$ for each step is provided. The KKT conditions (Eq.(7)) derived from the first order derivatives of $L$ in Eq.(5) is the foundation of conventional and on-line SVR formulation derivations.

\section{NUMERICAL EXAMPLES AND DIS- CUSSIONS}

The effectiveness of the proposed SVR-based approach for nonlinear structural identification is demonstrated by a five degree of freedom nonlinear structural identification problem, in which two cases (power parameter is known/unknown) are both investigated on the basis of the scheme in section 3 .
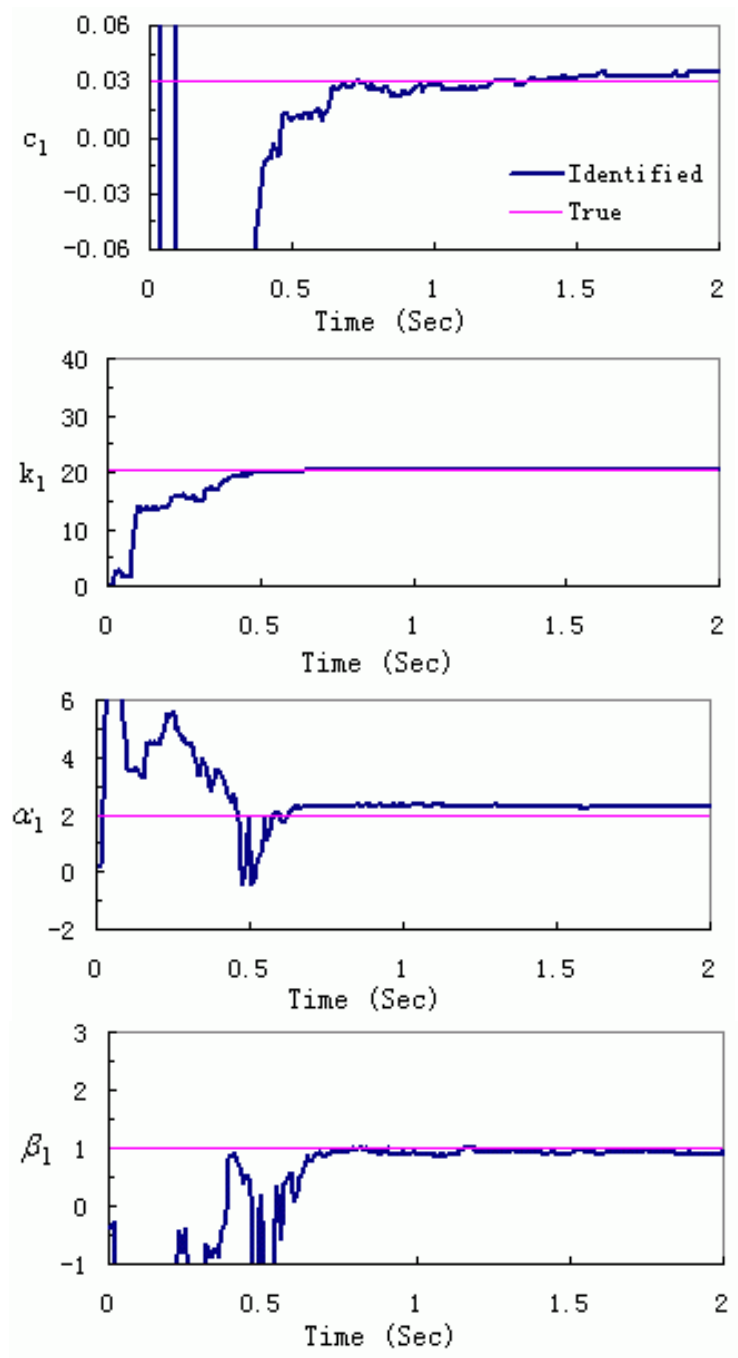

Fig.3 Identified results for first floor of 5-story hysteretic structure; $k$ in $\mathrm{N} / \mathrm{m}$ and $c$ in $\mathrm{N} / \mathrm{m} / \mathrm{s}$

\section{(1) Power parameter known case}

A 5-DOF nonlinear hysteretic structural system whose restoring forces are expressed by the versatile model is investigated here to make nonlinear structural identification. The mass, damping and stiffness of each floor of the 5-DOF system are, respectively, $m_{i}=0.12553 \mathrm{~kg}, c_{i}=0.03 \mathrm{~N} / \mathrm{m} / \mathrm{s}$ and $k_{i}=20.5 \mathrm{~N} / \mathrm{m}$. The structural parameters in Eq.(11) to control the restoring force nonlinear characters are $\alpha_{i}=2.0$, $\beta_{i}=1.0$ and $n_{i}=2$. The input seismic excitation is Kobe acceleration data adjusting the peak acceleration. It is necessary to amplify the selected ground excitation because amplified excitation provides sufficiently strong forces to bring the structure to the nonlinear range. The sampling interval of the structural responses is $0.01 \mathrm{~s}$. Assume that all the floor nonlinear seismic responses of the 5-DOF system are available, which are generated from the Runge-Kutta method numerically. To test the applicability of the proposed method in the presence of noise, 3\% standard white noises without frequency band limitation
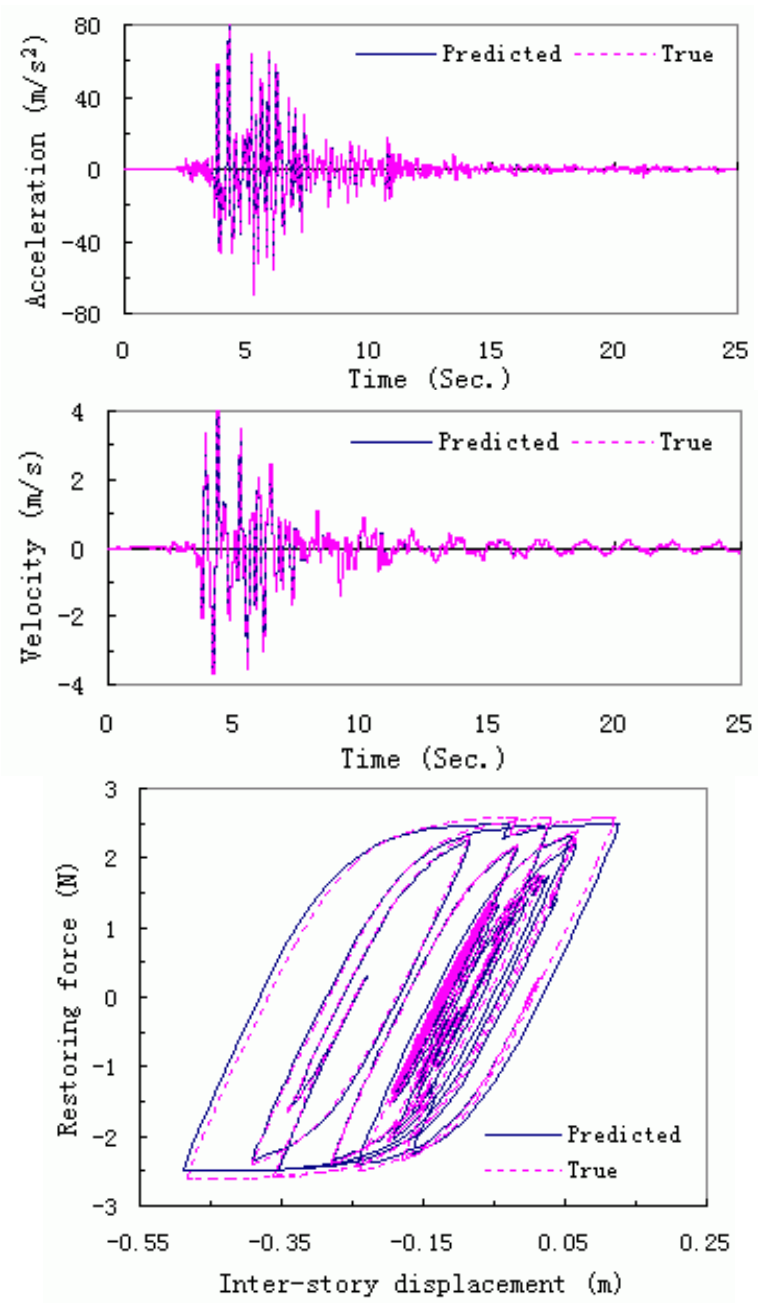

Fig.4 Comparisons of acceleration, velocity and hysteretic loop between re-simulated and observed values 
are added as observation noises, where $3 \%$ means the noise standard deviation is $3 \%$ of that of the simulated training data. Independent noises are added separately on each channel for each signal. It is significant to assume different noises on channels from the experimental point of view because each signal is measured from a different sensor. When the on-line SVR is used to identify the structural dynamic properties, special attentions should be given on how to choosing SVR parameters ${ }^{36)}$. SVR parameters mainly consist of $\bar{C}$ and $\varepsilon$. Parameter $\bar{C}$ determines the trade off between model complexity and the degree to which deviations larger than $\varepsilon$ are tolerated in the optimum formulation. A helpful equation is proposed by Cherkassky and $\mathrm{Ma}^{36)}$ :

$$
\bar{C}=\max \left(\left|\bar{y}+3 \sigma_{y}\right|,\left|\bar{y}+3 \sigma_{y}\right|\right)
$$

where $\bar{y}$ is the mean of the response, $\sigma_{y}$ the stan dard deviation of the training response value. $\varepsilon$ is a
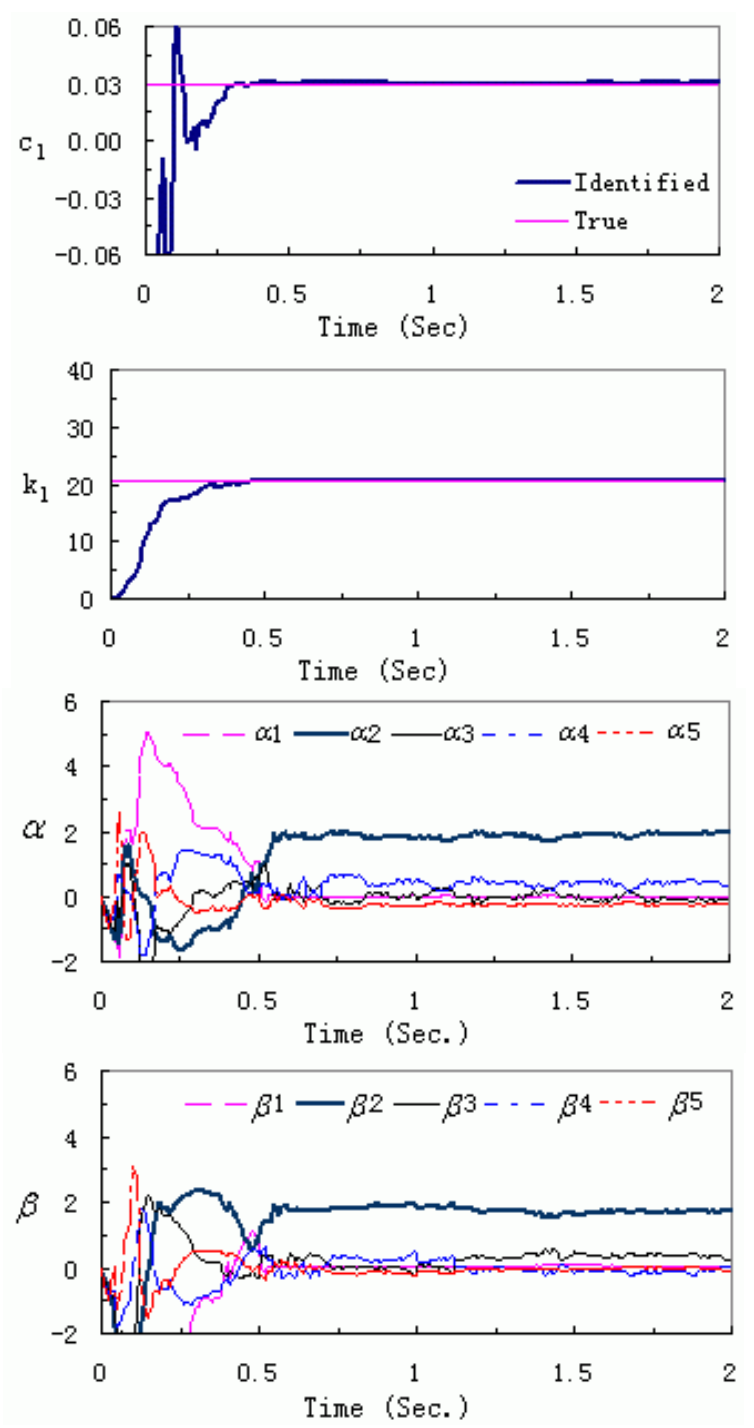

Fig.5 Identified results for first floor of 5-story hysteretic structure; $k$ in $\mathrm{N} / \mathrm{m}$ and $c$ in $\mathrm{N} / \mathrm{m} / \mathrm{s}$ parameter in the $\varepsilon$ insensitive loss function which controls the width of the $\varepsilon$ insensitive zone used to fit the training data. It is widely known that the selected $\varepsilon$ should reflect the noise level of the observed data. Cherkassky and $\mathrm{Ma}^{36}$ ) also recommend the following equation for choosing $\varepsilon$ :

$$
\varepsilon=\tau \sigma \sqrt{\frac{\ln N}{N}}
$$

where, $\sigma$ is noise standard variance, and $\tau$ should be tuned based on the data set sizes, noise levels and target functions. Cherkassky recommends that $\tau=3$ can give good performance for most cases $^{36)}$. The training data number should be chosen based on the trade off between computation time and the result accuracy, which is selected as $N=200$ here.

The mass of each floor is assumed known. By substituting the acceleration and velocity into Eq.(12), the restoring forces for each floor can be produced as observation data. In the implementation, SVR pa-
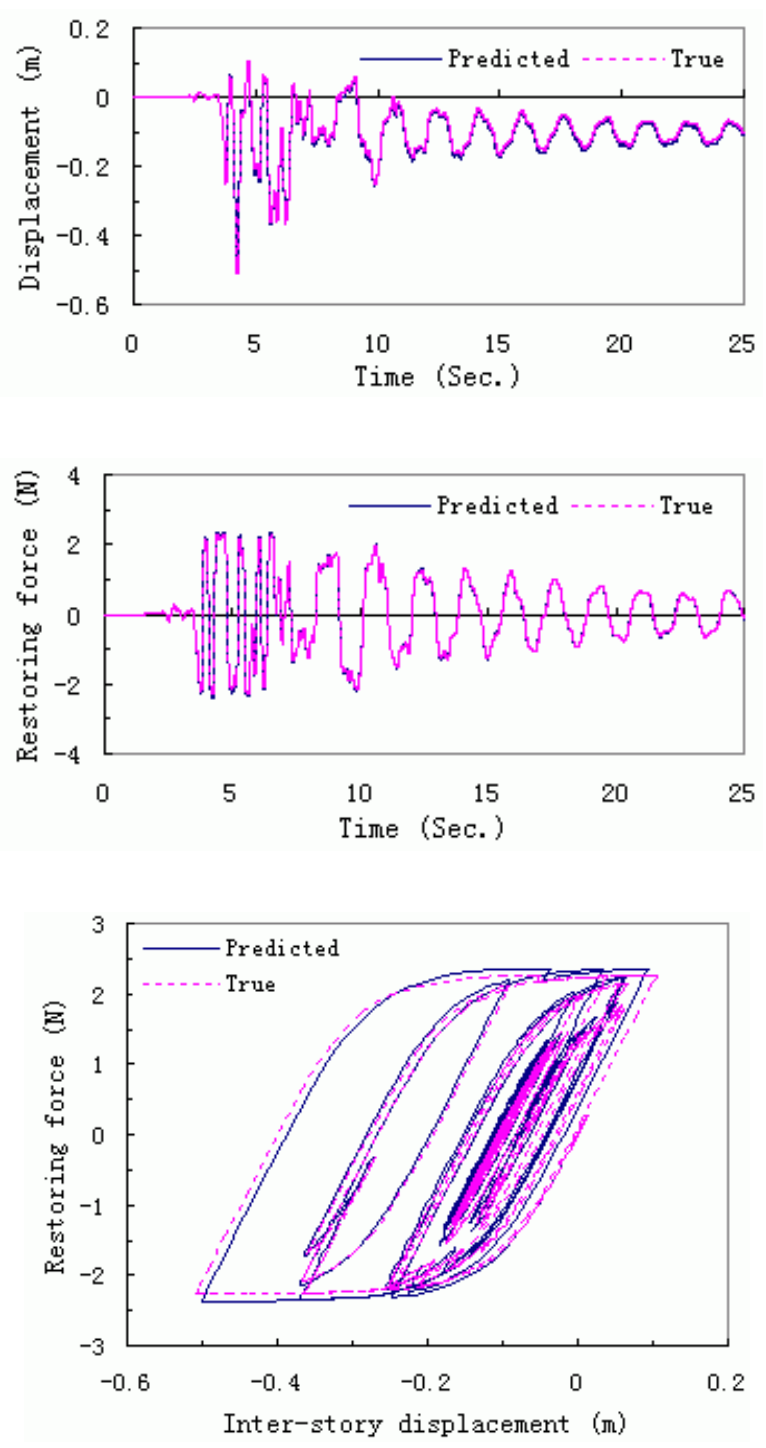

Fig.6 Resimulated and observed displacements, restoring forces and hysteretic loop between values 
rameters are chosen as $\bar{C}=10$ and $\varepsilon=0.3$. The time histories of the identified structural parameters and versatile model parameters of the first floor are illustrated in Fig.3. Good agreements between these parameters and the true values are evident, which shows that the proposed identification algorithm using the on-line SVR clearly is efficient for identifying nonlinear structural systems. Furthermore, previous work usng the SVR-based approach for structurtal identification has shown that the SVR-based approach can produced more accurate results than the least-square-based and the LS-SVM-based methods, especially in the non-Gaussian noide case ${ }^{26}$. It needs to note that the identified results at time $2 \mathrm{~s}$ have small offset errors, which are caused by the numerical error in the differential equation lineralization and the observation noise corrupted in the I/O noise. The re-simulated response of acceleration, velocity and hysteresis loop obtained from the identified parameters are provided in Fig.4 to compare with the true values in order to check the efficiency of the suggested approach.

\section{(2) Power parameter unknown case}

Generally, the power parameter $n_{i}$ in the Bounc-Wen model is unknown. In this case the structural response cannot be transformed to a linear form, and the presented on-line SVR-based structural identification approach do not succeed. A less exact but more practical strategy to overcome this shortcoming has been developed in section 3. Now we considers a five-story structural nonlinear model to verify the proposed on-line SVR-based approach in power parameter unknown case.

The five-story nonlinear building based on the Bounc-Wen model, each floor with the following characteristics: $m_{i}=0.12553 \mathrm{~kg}, c_{i}=0.03 \mathrm{~N} / \mathrm{m} / \mathrm{s}$, $k_{i}=20.5 \mathrm{~N} / \mathrm{m}, \alpha_{i}=2.0, \beta_{i}=2.0$ and $n_{i}=2$. The seismic excitation is Kobe earthquake data with peak value amplification. The sampling interval is $0.01 \mathrm{~s}$. As the observation noise, $1 \%$ standard white noise is added into the measurement data. In a similar procedure of the first case, the on-line SVR is executed in Matlab environment by choosing parameters $\bar{C}=50$ and $\varepsilon=0.01$. Fig.5 illustriates the on-line identified results for structural and versatile model parameters. Fig.5 clearly shows versatile model parameters $\alpha$ and $\beta$ corresponding to the $n_{i}=2$ have large values, and these two parameters corresponding to other false $n_{i}$ values are around zero, therefore the power parameter $n_{i}$ equals to 2 is identified indirectly. The comparisons of the identified response and hysteresis loop with the true values are shown in Fig.6. Agreement between these re-simulated results and the true values appears good, illustrating the efficiency of the developed linear identification algorithm for the structural system with versatile type of nonlinear dynamic characteristics even though the power parameter $n_{i}$ is unknown. As shown in Fig.6, the structure under seismic excitation vibrates around a negative values. This is an interesting phenomenon existing in civil engineering. A disadvantage of the proposed approach for $n_{i}$ unknown case is that it worked well in low noise coditions, however it fails to produce accurate results when noise level is high. Further work need to be studied for this problem. A possible way is adopting the model selection method based on Akaike-Bayes information Criterion (ABIC) ${ }^{37)}$ to identify the unknown power parameter directly.

\section{CONCLUSION}

A on-line SVR-based identification approach is proposed in this work to identify nonlinear structural parameters controlling the nonlinear structural constitutive performance. In particular, the case that power parameter of the Bouc-Wen model has been investigated by the proposed approach. In the literature, few approaches have been dedicated to this work. Numerical examples indicate that the suggested approach implements rapidly and works well due to the robust SVR utilized. Since the novel $\varepsilon$ insensitive loss function adopted and the "Max-Margin" idea used in SVR, the SVR-based approach identifies nonlinear structural parameters accurately, even when the I/O data are pollucted by noises. Moreover, an incremental training algorithm is employed to solve SVR formulations in a sequential way, which not only significantly reduces the computation time, but also makes the nonlinear structural identification on-line.

A more robust non-linear structural identification method is being studied to withstand higher-level noise pollucted in the I/O data, in which the unknown power parameter will be determined by a model selection strategy efficiently. Other topics also need further studies such as the time-varying structural identification problem.

\section{REFERENCES}

1) Hoshiya, M. : Structural identification by extended Kalman filter, J. Eng. Mech., Vol. 110, pp. 1757-1770, 1984.

2) Sato, T., and Qi, K. : Adaptive $\mathrm{H}_{\infty}$ filter: its application to structural identification, J. Eng. Mech., Vol. 124, pp. 
1233-1240, 1998.

3) Yoshida, I., and Sato, T. : Health monitoring algorithm by the Monte-Carlo filter based on non-gaussian noise. $J$. Natural Disaster Science, Vol. 24, pp. 101-107, 2002.

4) Yang, J. N., Yang, Y. L., Lin, S., and Huang, N. : Hilbert-Huang-based approach for structural damage detection, J. Eng. Mech., Vol. 130, pp. 85-95, 2004.

5) Bouc, R. : Forced vibrations of mechanical systems with hysteresis, Proc. of the Fourth Conference on Nonlinear Oscillations, Prague, 1967.

6) Wen, Y. K. : Method of radom vibration of hysteretic systems, J. Eng. Mech. Division, Vol. 102, pp. 249-263, 1976.

7) Iwan, W. D. and Gates, N. C. : Estimating earthquake response of simple hysteretic structures, J. Eng. Mech. Division, Vol. 105, No. 3, pp. 391-405, 1979.

8) Villaverde, R. and Lamb, R. C. : Scheme to improve numerical analysis of hysteretic dynamic systems, J. Struc. Eng., Vol. 115, No. 1, pp. 228-233, 1989.

9) Liu, J. L. : Exact solution of nonlinear hysteretic responses using complex mode superposition method and its application to base-isolated structures, J. Eng. Mech., Vol. 131, No. 3, pp. 282-289, 2005.

10) Distefano, N. and Rath, A. : Sequential identification of hysteretic and viscous models in structural seismic dynamics, Computer Methods in Applied Mech. and Eng., Vol. 6, Issue 2, pp. 219-232, 1975.

11) Sues, R. H., Mau, S. T. and Wen, Y. K. : System identification of degrading hysteretic restoring forces, J. Eng. Mech., Vol. 114, pp. 833-846, 1988.

12) Yar, M. and Hammond, J. K. : Parameter estimation for hysteretic systems, J. Sound and Vibration, Vol. 117, pp. 161-172, 1987.

13) Loh, C. H. and Chung, S. T. : A three-stage identification approach for hysteretic systems, Earthquake Eng. and Struc. Dynamics, Vol. 22, pp. 129-150, 1993.

14) Pei, J. S., Smyth, A.W. and Kosmatopoulos, E. B. : Analysis and modification of Volterra/Wiener neural networks for the adaptive identification of nonlinear hysteretic dynamic systems, J. Sound and Vibration, Vol. 275, pp. 693-718, 2004.

15) Saadat, S., Buckner, G. D., Furukawa, T. and Noori, M. : An intelligent parameter varying (IPV) approach for nonlinear system identification of base excited structures, Int. J. Nonlinear Mech., Vol. 39, Issue 6, pp. 993-1004, 2004.

16) Tan, R. Y. and Weng, I. W. : Identification of dynamic properties of isolated structures, Eng. Struc., Vol. 18, Issue 3, pp. 240-246, 1996.

17) Benedettini, F., Capecchi, D. and Vestroni, F. : Identification of hysteredc oscillators under earthquake loading by nonparametric models, J. Eng. Mech., Vol. 121, Issue 5, pp 606-612, 1995.

18) Worden, K. K. and Panet, M. : Identification of hysteretic systems using the differential evolution algorithm, $J$. of Sound and Vibration, Vol. 248, Issue 2, pp.289-314, 2001.

19) Li, S. J., Suzuki, Y. and Noori, M.: Identification of hysteretic systems with slip using bootstrap filter, Mech. Systems and Signal Processing, Vol. 18, Issue 4, pp. 781-795, 2004.
20) Ni, Y. Q., Ko, J. M. and Wong, C. W. : Identification of nonlinear hysteretic isolators from periodic vibration tests, J. of Sound and Vibration, Vol. 217, Issue 4, pp. 737-756, 1998

21) Jann, J.N., Yang, N. and Lin, S. : On-line identification of nonlinear hysteretic structures using an adaptive tracking technique, Int. J. of Nonlinear Mech., Vol. 39, Issue 9, pp. 1481-1491, 2004.

22) Smyth, A. W., Masri, S. F., Chassiakos, A. G. and Caughey, T. K. : On-line parametric identification of MDOF nonlinear hysteretic systems, J. Eng. Mech., Vol. 125, No. 2, pp. 133-142, 1999.

23) Vapnik, V. N. : The Nature of Stastical Learning Theory, Second edition, Spring, 1999.

24) Suykens, J. A. K. and Vandewalle, J. : Least squares support vector machine classifiers, Neural Process Letters, Vol. 9, pp. 293-300, 1999.

25) Gunn, S. R. : Support vector machines for classification and regression, Technical Report, Univ. of Southampton, 1998.

26) Rousseeuw, P.J. and Leroy, A.M.: Robust Regression and Outlier Detec-tion, Wiley, New York, 1987.

27) Evgeniou, T., Pontil, M. and Poggio, T. Regularization networks and support vector machines, Advances in computational mathematics, Vol. 13, pp. 1-50, 2000.

28) Sato, T. and Zhang, J. : Online Identification of Large-Scale Structures with Robust Support Vector Regression, the secont international workshop on advanced smart materials and samert structures technology, Korea, 2005.

29) Shampine, L. F. : Numerical Solutions for Ordinary Differential Equations, Chapman and Hall, 1994.

30) Osuna, E. E., Freund, R. and Girosi, F. : Support vector machines: training and applications, Technical Report, Massachusetts institute of technology, March, 1997.

31) Platt, J.C. : Sequential minimal optimization: a fast algorithm for training support vector machines, Technical Report MSR-TR-98-14, Microsoft Research, April, 1998.

32) Flake, G.W. and Lawrence, S. : Efficient SVR regression training with SMO, Machine Learning, Vol. 46, pp. 271-290, 2002.

33) Cauwenberghs, G. and Poggio, T. : Incremental and decremental support vector machine learning, in: Advance in Neural Information Processing Systems, Vol. 13, Cambridge, MIT Press, pp. 409-423, 2001.

34) Ma, J., Theiler, J. and Perkins, S. : Accurate on-line support vector regression, Neural Computation, Vol. 15, pp. 2683-2703, 2003.

35) Martin, M. : On-line support vector machines for function approximation, Technical Report LSI-02-11-R, Software Department, University of Politecnica Catalunya, Spain, 2004.

36) Cherkassky, V. and Ma, Y. : Practical selection of SVM parameters and noise estimation for SVM regression, Neural Networks, Vol. 17, pp. 113-126, 2004.

37) Akaike, H. : Likelihood and Bayes Procedure with Discussion, Valencia Univ. press, 1980.

(Received June 17, 2005) 